\title{
Programmable Metamaterials for Software-Defined Electromagnetic Control: Circuits, Systems, and Architectures
}

\author{
Sergi Abadal ${ }^{\circledR}$, Member, IEEE, Tie-Jun Cui ${ }^{\circledR}$, Fellow, IEEE, Tony Low, \\ and Julius Georgiou ${ }^{\circledR}$, Senior Member, IEEE
}

\begin{abstract}
Metamaterials and their two-dimensional analogues, metasurfaces, have recently attracted enormous attention because of their powerful control over electromagnetic (EM) waves from microwave to visible range. Moreover, by introducing explicit control of its sub-wavelength unit cells, a metamaterial can become programmable. Programmable metamaterials may not only host multiple EM functionalities that can be chosen or combined through simple software directives, but also be provided with means to adapt to the environment or communicate with other metamaterials, thereby enabling a myriad of applications in sensing, imaging, or communications. The realization of such a software-driven cyber-physical vision comes, however, at the cost of significant hardware requirements. In this paper, recent progress in the field of programmable metasurfaces is reviewed, cutting across layers from the application down to the device and technology levels. The main aim is to present the current status, main benefits, and key challenges of this thriving research area with a tutorial spirit and from a hardware perspective.
\end{abstract}

Index Terms-Programmable metamaterials, programmable metasurfaces, software-defined electromagnetic control, cyberphysical systems, embedded digital systems.

\section{INTRODUCTION}

$\mathbf{M}$ ETAMATERIALS are artificial structures that enable the realization of novel electromagnetic (EM) components with engineered and even unnatural functionalities. Through the unprecedented control over electromagnetic waves, achieved thanks to the manipulation of impedance at subwavelength scale, metamaterials have led to significant breakthroughs in fields such as imaging, radar, or wireless communications [1]-[4] across the whole spectrum [5]-[10].

Manuscript received January 27, 2020; revised February 16, 2020; accepted February 18, 2020. Date of publication February 24, 2020; date of current version March 12, 2020. This work was supported by the European Commission under Grant H2020-736876-VISORSURF, and in part by the National Key Research and Development Program of China under Grant 2017YFA0700201, Grant 2017YFA0700202, and Grant 2017YFA0700203. This article was recommended by Guest Editor A. Wu. (Corresponding author: Sergi Abadal.)

Sergi Abadal is with the NaNoNetworking Center in Catalonia (N3Cat), Universitat Politècnica de Catalunya, 08034 Barcelona, Spain (e-mail: abadal@ac.upc.edu).

Tie-Jun Cui is with the School of Information Science and Engineering, Southeast University, Nanjing 210096, China (e-mail: tjcui@ seu.edu.cn).

Tony Low is with the Department of Electrical and Computer Engineering, University of Minnesota, Minneapolis, MN 55455 USA (e-mail: tlow@umn.edu).

Julius Georgiou is with the Department of Electrical and Computer Engineering, University of Cyprus, 1678 Nicosia, Cyprus (e-mail: julio@ucy.ac.cy).

Color versions of one or more of the figures in this article are available online at https://ieeexplore.ieee.org.

Digital Object Identifier 10.1109/JETCAS.2020.2976165
However, due to their resonant nature, most existing metamaterials have been typically tailored for a single application working under preset conditions (e.g. electromagnetic cloaking for a fixed angle of incidence and at a particular frequency) and cannot be reused. This clearly limits their applicability and, considering the complexity of the current metamaterial design processes, increases their cost.

In response to these limitations, recent years have witnessed the advent of different types of tunable and reconfigurable metamaterials [11], [12]. Reconfigurability is achieved by using components that tune the metamaterial response or that completely change the current flows within the metamaterial, including varactors or variable resistors [13], PIN diodes or switches [14], microelectromechanical systems (MEMS) [15], [16], mechanical parts [17], or advanced materials such as graphene [18], [19] or liquid crystals [20].

A natural step further in this domain has been the proposal of the programmable metamaterial paradigm. To achieve programmability, metamaterials need to be provided with ways to control the components that realize the reconfigurability. Current developments generally account for a Field Programmable Gate Array (FPGA) that drives an appropriately digitized version of a reconfigurable metamaterial [14], [21], [22]. An evolution of this approach has been envisaged recently, where the external FPGA is replaced by a network of tiny sensors and controllers that are integrated within the structure of the metamaterial and interfaced with the real world [23], [24]. The ultimate goal of this cyber-physical system vision is to turn metamaterials into software-driven, intelligent, autonomous yet interconnectable devices capable of hosting multiple functionalities and using them while adapting to the needs of the system.

Fundamentally, the behavior of programmable metamaterials hinges on the collective response of the unit cells. This, effectively, represents a discretization of both the space (with subwavelength granularity) and the response of each unit cell (with a discrete set of amplitudes and phases). Due to their inherent "digital" nature, programmable metamaterials have been associated to the coding metamaterial paradigm where the device is encoded using a discrete set of unit cell states. The state of the metamaterial can be thus elegantly described as a state matrix, thus providing a powerful and intuitive design perspective while drawing a clear parallelism with information theory. This opens new ways to model, compose, and design metamaterials [25]-[28], and has proven helpful in tackling the challenge of packing multiple functionalities within a single device and serving multiple purposes concurrently.

The cyber-physical view of programmable metamaterials, with the added properties of intelligence and interconnectivity, 


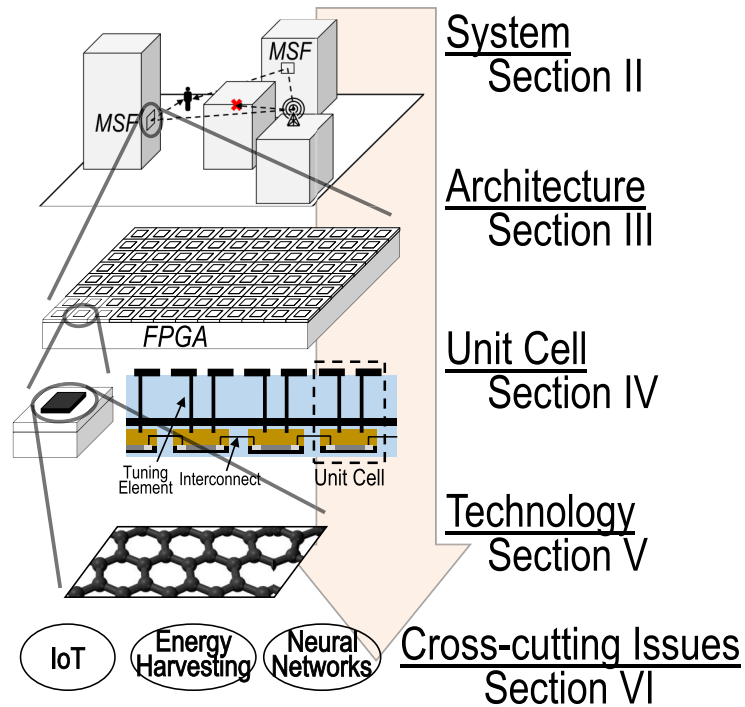

Fig. 1. Graphical abstract of this tutorial paper. Applications demand the deployment and use of programmable metasurfaces, whose architecture includes a number of tunable unit cells. Unit cells integrate different components, materials, and technologies. Finally, several cross-cutting paradigms can impact the design of the whole system.

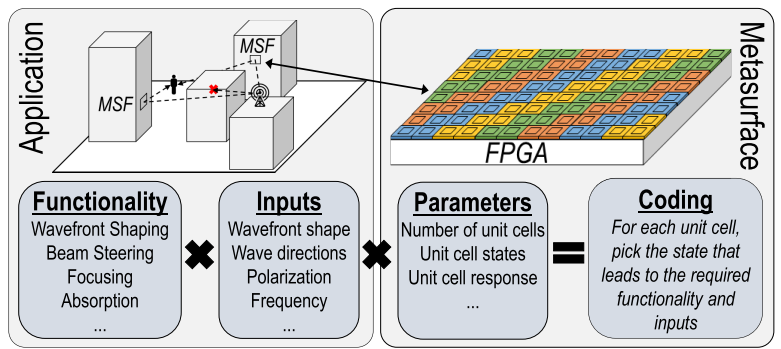

Fig. 2. From the application to the metasurface coding: applications require the use of multiple functionalities (e.g. beam steering) with programmable inputs (e.g. direction of reflected waves) that, depending on the metasurface characteristics, lead to a particular metasurface coding. Coding refers to the assignment of states to unit cells to achieve the target functionality and inputs as accurately as possible. Any change to the functionality or inputs leads to a different coding, hence the need for programmability.

is expected to open the door to a plethora of applications in areas such as sensing, communications, or imaging [12], [29]-[31]. A good example in communications would be the concept of programmable wireless environment, which aims to revolutionize wireless communications with significant impact in 5G networking and AR/VR applications [32], [33]. In imaging, the analogy between the unit cells of a programmable metamaterial and the pixels of a screen allow to envisage practical ways to create dynamic holograms [22]. Further, implementing programmable metamaterials in the terahertz (THz) band could aid in closing the so-called terahertz gap, with important implications in imaging or wireless communications applications [9], [34], [35].

While the potential of the programmable metamaterials is high, harnessing software-defined electromagnetic control poses significant challenges at all levels, from the system down to the circuit or the technology. For these devices to be practical, the associated control circuits must comply with stringent limitations in terms of area, power consumption, or interference. At the same time, the control system needs to be powerful enough to cover a representative pool of potential electromagnetic functionalities and applications. This imposes severe restrictions on the practicable design space of circuits and systems, thus encouraging the proposal of smart, metamaterial-aware and opportunistic, highly streamlined solutions at the circuit, system, and full architecture levels. In a longer term, the use of emerging technologies such as graphene, nanoscale integrated sensors, opportunistic energy harvesting, or neural networks for metamaterial control may be a critical enablers of fully autonomous programmable metamaterials.

This paper, as well as the special issue where it belongs to, provides an overview of the research avenue of programmable metamaterials and describes recent advances from a hardware perspective. We cover the areas ranging from tunable metamaterial engineering, low-power circuits and systems design, nanoelectronics, and system architecture, encompassing both contributions close to production and more prospective proposals with longer-term potential.

The paper is organized in a top-down approach. First, in Section II, the fundamentals of programmable metamaterials are reviewed from the system perspective, delving into the prospects and applications of such systems. In Section III, recent trends are surveyed from the architectural standpoint, analyzing the existing FPGA-based and fully integrated approaches. Next, in Section IV, we focus on challenges and advances at the component and circuit levels for practical computation, communication, sensing, and tuning at the unit cells. In Section V, we dive deeper down to the technology, emphasizing novel materials, fabrication methods, and integration approaches allowing to extend the programmable metamaterial paradigm towards $\mathrm{THz}$ and optical frequencies. Before concluding, cross-cutting issues and advanced topics such as energy harvesting, approximate computing, or the application of neural networks to coding metamaterials are discussed in Section VI. Finally, the paper is concluded in Section VII.

\section{A Primer on Programmable Metasurfaces: OUTLOOK AND APPLICATIONS}

Programmable metasurfaces, as the thin-film counterpart of programmable metamaterials, serve to exemplify the process from the application to the design and coding of the device. Such a process is illustrated in Fig. 2 and developed herein. We note that, although similar concepts can be applied to 3D metamaterials, issues related to fabrication complexity, losses, cost, or bulkiness encourage the use of metasurfaces (i.e. metamaterials with subwavelength thickness) instead [36].

Metasurfaces enable a new set of applications (Sec. II-A) emerging from the unique and programmable control of the EM response. In essence, an application requires the metasurface to implement a certain EM functionality (Sec. II-B) with a given set of inputs. The metasurface is then encoded to that end, this is, the unit cells are set to the states that lead to the targeted functionality and inputs with the least possible deviation (Sec. II-C). The ultimate aim of the programmable metamaterials field is to be able of doing so with the minimum effort, being able to automatically adapt to varying conditions over time, with little to no power consumption, among other desired properties listed in Section II-D.

\section{A. Emerging Applications}

By virtue of the unique real-time reconfigurability of programmable metasurfaces, many novel ideas and solutions have been introduced into traditional industries with numerous emerging applications appearing. Additionally, extending the 


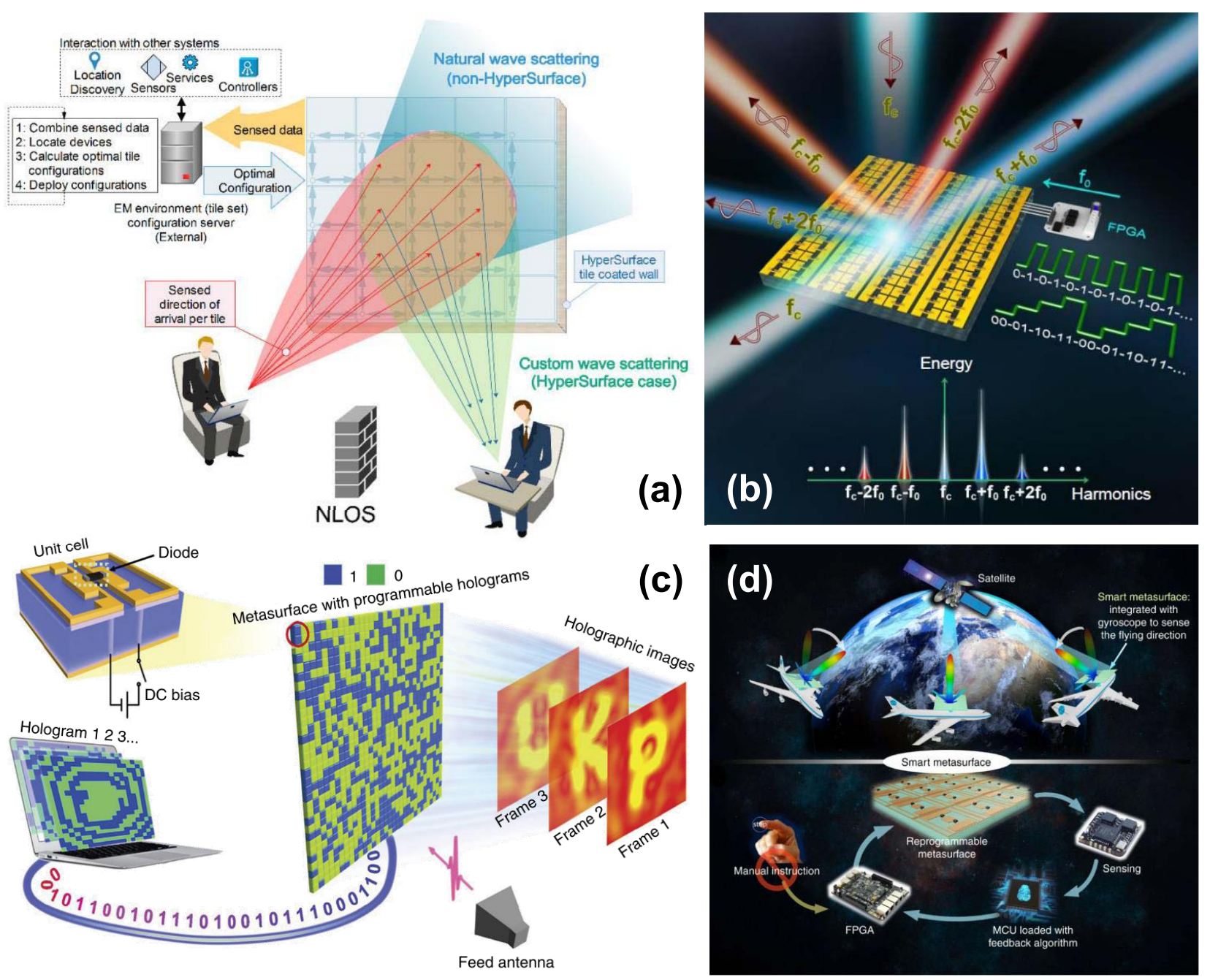

Fig. 3. Emerging applications enabled by the programmable metamaterial approach, including (a) programmable and self-adaptive wireless environments [37], (b) simplified architectures for multi-channel wireless transceivers [38], (c) holographic images [22], and (d) self-adaptive smart metasurfaces [30].

use metasurfaces in the millimeter-wave (mmWave) and $\mathrm{THz}$ bands is expected to open the door to multiple opportunities across multiple fields. Here we list some of these applications, emphasizing the key role of real-time reconfigurability and the potential of mmWave-THz solutions when appropriate.

1) Wireless Communications in $5 G$ and Beyond: The field of wireless communications is seeing a revolution in the use of programmable metasurfaces as intelligent reflecting surfaces. This enables the concept of programmable wireless environments as seen in Fig. 3(a). A wireless environment is defined as a group of physical objects that can significantly alter the propagation of EM waves among communicating devices [32], [37], [39]-[41]. In general, the environment shows a negative effect on the communication efficiency due to the energy loss from material absorption and free-space propagation, and the multipath effects caused by EM wave scattering from the diffraction, reflection, and refraction phenomena. However, the emergence of programmable metasurfaces featuring EM-wave re-engineering enables the implementation of programmable wireless environments. By introducing different functions like focusing, scattering and steering at selected spots and at different rates, the programmable metasurfaces make the wireless environment capable of handling the EM propagation in a manner reminiscent of routers and firewalls in classical networking, which mitigate both propagation loss and multipath effects existing in the current wireless communication systems. This is of special importance in the mmWave and $\mathrm{THz}$ bands, where high spreading losses and the inability of EM waves to penetrate walls and most objects creates a distance problem [42]. In this application context, especially at high frequencies, the main challenge is to develop metasurfaces that offer fine-grained programmability but, at the same time, are cost-efficient and scalable to relatively large walls. Otherwise, lower cost alternatives to the same problem (e.g. using active relays) will probably be adopted instead [43].

Another application related to wireless communications is the development of simplified architectures for wireless communication transmitters. In the traditional wireless communication transmitters, both in the heterodyne or homodyne architectures, the baseband signal processing module and the radio-frequency (RF) chain module are separated from each other. The information is modulated in the baseband and then up-converted to the RF frequency through the RF chain. As more and more protocols and standards are proposed to satisfy the increasing demands of stability, high speed and low latency in the information era, the RF components are facing higher complexity requirements in design and manufacturing, resulting in extremely high hardware cost and energy consumption in practical implementation. Recently, several simplified wireless-communication transmitter architectures based on programmable metasurfaces have been proposed with low 
hardware complexity, high cost effectivity, and good performance [38], [44]. As shown in Fig. 3(b), the metasurfaces are utilized to directly modulate the carrier wave in multiple channels using the baseband signal without the need of core components of the RF chain such as mixers, filters, amplifiers, etc. At high frequencies, this is crucial as these components can be lossy and significantly complex. In any case, the required refresh rate of the metasurface is thus the modulation rate. Although the principle is similar to that of direct antenna modulation (DAM) technology [45], the programmable metasurface approach possesses better performance and higher efficiency due to more flexible tunability of EM waves. The novel architecture has shown great potential to bring a new paradigm that naturally integrates the signal processing algorithms in information science and the hardware of the programmable metasurfaces for future-generation wireless communications. Eventually, a great challenge will be to prove whether simplified transceiver architectures, together with metasurfacebased steering mechanisms, lead to mmWave- $\mathrm{THz}$ chipsets that, in contrast to classical architectures, are practical enough for their take up in future wireless communication systems.

2) Holographic Imaging: Holography is one of the most promising imaging techniques that attract tremendous attention covering many applications in display, security, data storage, etc. However, most of the conventional holograms suffer from low resolution, limited image quality, and fixed holographic image. The programmable metasurface-based holograms, illustrated in Fig. 3(c), feature two major advantages compared with the conventional holograms [22]. Firstly, the outstanding recording ability for both amplitude and phase information of wavefront in the subwavelength scale provide excellent spatial resolution. Secondly, the pixel (i.e. the metasurface unit cell) level programmability greatly contributes to realtime and arbitrary holographic images. Metasurfaces should thus be programmable at typical refresh rates of displays, this is, around 60 frames per second. If achieved, these remarkable properties make the programmable metasurface one of the competitive candidates in the future holographic imaging and even holographic movies.

3) Self-Adaptive Systems: Recently, researchers have pursued the implementation of intelligent metasurfaces, which are self-adaptive to control the presented functions by the metasurface itself [30], [46]. As shown in Fig. 3(d), the programmable metasurface constructs a closed-loop system that, in collaboration with some specific sensors and feedback circuits, can operate on its own without human intervention. The speed at which the metasurface needs to be updated will depend on the end-user application and the main challenge resides in the building of the lightweight, robust, real-time control algorithms that will close the loop. In any case, such systems are expected to open up a new avenue for future unmanned devices and systems that are consistent with the ambient environment, which further pave the way towards intelligent and cognitive metasurfaces.

\section{B. Electromagnetic Functionalities}

Digital coding and programmable metamaterials can be applied to realize diverse EM functionalities due to their advantages. By changing the coding matrices pre-stored in an FPGA, many functionalities can be switched in real time, leading to many promising applications. In fact, the possibility of manipulating the response in the time domain opens the door to multiple new opportunities. Next, we briefly describe a comprehensive set of EM functionalities implemented by

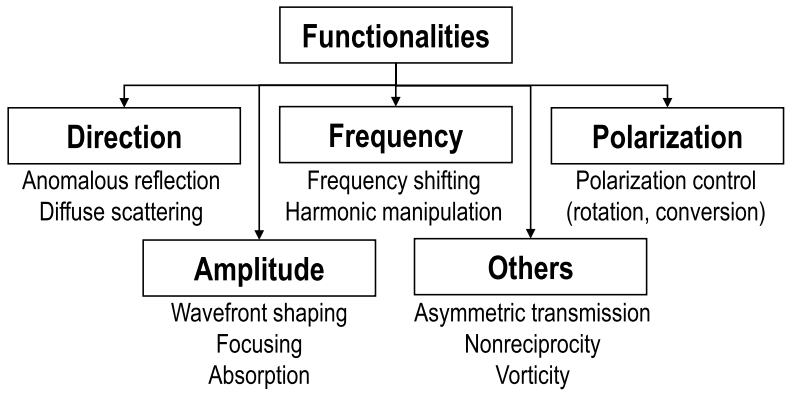

Fig. 4. Summary of electromagnetic functionalities achievable with programmable metasurfaces. Note that, depending on the unit cell structure, such functionalities can be achieved in reflection, transmission, or both. Programmable metasurfaces can also host multiple functionalities, implement distributed intelligence, and react to the environment.

coding and programmable metamaterials in the literature, classified in Figure 4.

- Wavefront shaping. Metasurfaces have shown their powerful capabilities in engineering the EM wavefronts. Coding and programmable metasurfaces have the advantages of simplifying the design procedures and of switching among different functions in real time, which can be used to shape both reflection and transmission wavefronts on a shared aperture [47]. Furthermore, the full-space wavefront shaping can be dynamically switched between reflection or transmission modes by changing the PIN diodes states [48].

- Focusing. Programmable metasurfaces have also been used to focus the EM fields. By applying the speciallydesigned coding matrices, a programmable metasurface can be used to generate arbitrary focusing points dynamically, and field intensity at each point can also be controlled independently [49]. Besides, a programmable Huygens' metasurface was realized to focus the transmitted fields dynamically with high efficiency [50].

- Anomalous reflection. Metasurfaces can introduce an abrupt phase shift on the interface by designing the corresponding unit cell, which will result in phase discontinuities. Based on the idea of generalized Snell's laws, the programmable metasurface can realize anomalous reflection by using the phase-gradient space-coding sequence [51].

- Asymmetric transmission. Metamaterials can be used to realize the asymmetric transmission by using chiral structures. Programmable chiral metamaterials integrated with PIN diodes have been proposed to achieve dynamic control of the asymmetric transmission by changing the control voltages [52].

- Polarization control. Polarization is a very important dimension of EM waves. How to control the polarization state has always been the concern of researchers. Programmable metasurfaces also have an outstanding ability in controlling the polarization, which can be used for reconfigurable linear polarization conversion [14]. Furthermore, the varactors-based programmable metasurface can be dynamically switched among the functionalities of linear-to-linear, linear-to-elliptical, and liner-to-circular polarization conversion [53].

- Vorticity. Vortex waves carrying orbital angular momentum (OAM) can be used for orthogonal multiplexing communication via different OAM modes, which has the feature of helical wavefront and the property of mode orthogonality. Recently, coding metasurfaces were 
developed to generate vortex beams with different modes, including spin-controlled vortex beams [54].

- Absorption. Metamaterials have been widely studied for absorption in the past decade. Most metamaterial absorbers are designed in a fixed frequency band. Programmable metamaterials can be utilized to absorb the incident waves at different frequencies by electrically controlling the biasing voltages on the varactors [55], which can extend the working frequency range of absorbers.

- Diffuse scattering. By optimizing the coding pattern, coding metasurfaces can be used for scatteringsignature reduction by the strategy of diffuse scattering in both microwave and terahertz regime [56]. The recently proposed space-time-coding modulation in programmable metasurfaces can also redistribute the scattered power in both space and frequency domains uniformly, thereby leading to unprecedented levels of Radar Cross-Section (RCS) reduction [28].

- Frequency shifting. By modulating the metasurface properties in the time domain, a programmable metasurface can control the reflected waves in the frequency domain. For instance, frequency shifting can be realized by imparting suitable time modulation signals on programmable metasurface, such as frequency conversion [38], and harmonic frequency generation [28].

- Nonreciprocity. By introducing the time-varying approach, the space-time-coding programmable metasurface was successfully used to break reciprocity which relied on inducing suitable spatiotemporal phase gradients in a programmable way via digital coding modulation [28], [57]. Besides, the amplifier transistors-based programmable metasurface was also proposed to realize forward and backward nonreciprocal transmissions, but without using the time-varying modulation [58].

It is worth noting that, depending on the unit cell structure, the functionalities are achieved either in reflection [18], [56] or transmission [59], [60]. Specific designs may support both modes simultaneously [47] or switch among them [48].

\section{Metamaterial Coding}

Coding and programmable metamaterials describe the EM performance from the information aspect, providing us novel and brief approaches to design functional metamaterials [21], [36]. The process of coding consists in assigning a particular state to each unit cell of the metasurface among the set of possible states, so that final coding pattern attains the targeted functionality with the least error. For instance, let us consider a unit cell designed to take four discrete values. Thus, the coding set is $\Sigma=\left\{s_{0}, s_{1}, s_{2}, s_{3}\right\}$. Depending on the desired functionality, different strategies can be used for the mapping. Here, we briefly summarize the main aspects involved in the coding of programmable metamaterials, as illustrated in Fig. 5.

1) The Coding States of the Unit Cells: The coding states of the unit cells are usually employed to represent the EM coefficients, which are the fundamental factors when designing the coding and programmable metamaterials. There are two major coding schemes:

- Phase coding states. The phase coding scheme is the most common one in coding and programmable metamaterials. By adopting two unit cells with different geometries or introducing PIN diodes in the single unit cell with dynamical modes [21], the reflected or transmitted phase

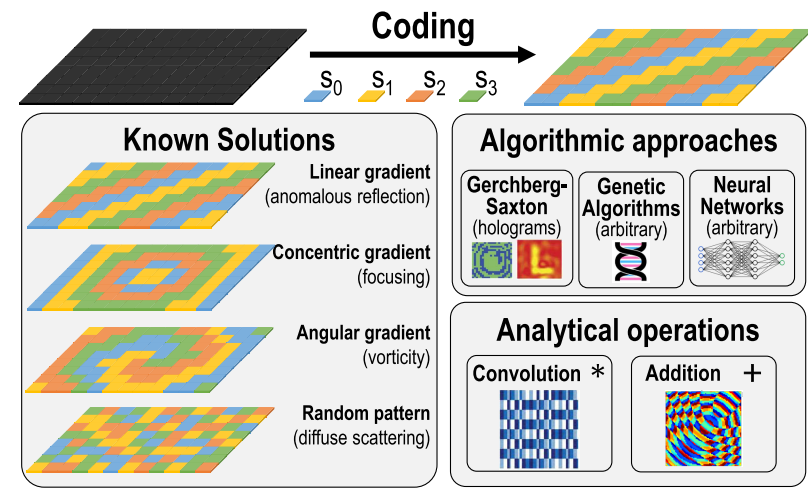

Fig. 5. Illustration of the coding of a metasurface with a set of four states and existing methods to encode a metasurface for different functionalities.

responses satisfy the conditions for the 1-bit and multi-bit digital states. These concepts can also be promoted to polarization- or frequency-dependent coding metamaterials to form multi-channel coding strategies [61], [62].

- Amplitude coding states. The coding principle also enables digital descriptions of amplitudes, which can control the radiating power by using indium tin oxide (ITO) films or amplifiers [63]. A combination of amplitude and phase coding further enables full-space radiation control, in which the incident polarizations or PIN diodes are utilized for the real-time modulations for the amplitude coding states [48].

2) The Fundamental Methods to Map Metasurface States to Specific Functionalities: Due to the digital expressions of EM properties, the EM functionalities of coding and programmable metamaterials are decided by the distributions of the coding states, namely the coding patterns. Some of the coding patterns rely on known, often analytical solutions, derived from fundamental rules such as the generalized Snell's laws of reflection. Such common coding patterns are the following:

- Linear phase gradient for anomalous reflection and transmission. The directions of the scattering waves can be controlled by forming linear phase gradient coding patterns on the interfaces, according to the generalized Snell's laws of reflection [64]. The higher the number of unit cell states, the more anomalous manipulations (and even negative reflection) can be achieved [60], [65]. The detailed deflecting angle also relies on the period of the phase gradient and the working wavelength [61].

- Concentric phase gradient for focusing and beam forming. When the coding states are arranged in concentric phase gradient, the incident planar EM wave can be focused to a certain spotlight. The focal length depends on the period of the phase gradient, whereas the lateral position of the focal point is dependent on the position of the center of the concentric gradient [19]. Inversely, this coding pattern is also capable to form pencil beams in far field by converting the spherical waves to planar waves [14].

- Angular gradient for vortex beams. The phase coding states in angular gradient enable the radiations of vortex beams. The digital states with single or several periods of $0-2 \pi$ are placed spirally on the aperture, clockwise or anticlockwise, to construct different OAM modes. [54].

- Random pattern for RCS reduction. By establishing the coding states in random patterns other 
than the abovementioned gradient cases, the input power is reflected to arbitrary directions. Hence, the RCS is reduced notably [66]. Several optimization methods including particle optimization, genetic algorithms, or Golay-Rudin-Shapiro (GRS) polynomials model have also been introduced into the coding pattern design and accomplished almost homogeneously anomalous reflection [67].

The methods above are powerful, but do not generalize to complex functionalities or arbitrary wavefronts. To that end, several alternatives have arisen to facilitate the programming of metasurfaces. Besides the use of analytical operations on the coding patterns, explained in the subsection below, we find that programmable metasurfaces are amenable to widely used algorithms and optimization techniques, namely:

- Gerchberg-Saxton algorithm for holograms. Numerical algorithms such as Gerchberg-Saxton (GS) allow to build arbitrary holograms for near-field imaging and information transmission [22], [49]. Especially in the programmable designs, the holograms are also reprogrammable, indicating that the multiple desired holographic images can be realized in real time.

- Optimization algorithms and neural networks. To achieve arbitrary complex wavefronts that cannot be described as the combination of the patterns above, classical optimization algorithms can be employed. Evolutionary methods such as genetic algorithms or particle swarm optimization have been applied in several works [14], [66]. Further, the use of neural networks as a powerful tool to program and control metasurfaces is an emerging possibility (see Section VI for more details).

3) Analytical Operations on Multiple Coding Patterns: The methods described above can achieve a comprehensive set of coding patterns for multiple functionalities. If more advanced functionalities are desired, more complicated analytical operations should be implemented on multiple coding patterns. The following two methods allow to build complex coding patterns as the combination of two or more simpler patterns, using intuitive mathematical formalisms:

- Convolution operations. The purpose of convolution operations is to realize more precise EM control, breaking through the limitation of deflecting angle from the period of the phase gradient and the working wavelength. Convolution operations perform Fourier transformations on coding and programmable metamaterials, allows steering of the scattering pattern to an arbitrarily pre-designed direction only through the modulus of several coding patterns. Theoretically, the 1D and 2D convolution operations permit the radiating beams to radiate in the whole half space [26].

- Addition theorem. In fact, the essence of convolution operations is the phase multiplication of the coding states. The other operation, addition starts from the basic EM theorem and originally involves all the complex phase parts $e^{j \varphi}$ for their addition, implying the final result would contain all the added components. Hence, it means a superposition of the coding patterns, i.e., the corresponding scattering patterns and functionalities can appear simultaneously on the shared aperture [27], [68].

\section{Desired System Properties}

The methods described in the previous section have allowed to achieve a wide range of functionalities. There has been,
TABLE I

Representative Programmable Metasurfaces in the Literature

\begin{tabular}{|c|c|c|c|c|c|}
\hline Functionality & Approach & Freq. & Bits & Year & Ref \\
\hline \hline Absorption & Analytical & $5 \mathrm{GHz}$ & N/A & 2013 & {$[55]$} \\
\hline Low scattering & PSO & $10 \mathrm{GHz}$ & 3 -bit & 2014 & {$[66]$} \\
\hline Low reflection & PSO & $1.5 \mathrm{THz}$ & 3 -bit & 2015 & {$[56]$} \\
\hline Multi-function & GA & $10 \mathrm{GHz}$ & 1 -bit & 2016 & {$[14]$} \\
\hline Imaging & Analytical & $10 \mathrm{GHz}$ & 2 -bit & 2016 & {$[59]$} \\
\hline Beam steering & Convolution & $1 \mathrm{THz}$ & 2 -bit & 2016 & {$[26]$} \\
\hline Hologram & GS & $8 \mathrm{GHz}$ & 1 -bit & 2017 & {$[22]$} \\
\hline Focusing & Analytical & $0.2 \mathrm{THz}$ & 3 -bit & 2017 & {$[69]$} \\
\hline Vorticity & Convolution & $15 \mathrm{GHz}$ & 3 -bit & 2017 & {$[54]$} \\
\hline Multi-function & Analytical & $1.5 \mathrm{THz}$ & 3 -bit & 2017 & {$[70]$} \\
\hline RCS reduction & SA & $12 \mathrm{GHz}$ & 1 -bit & 2018 & {$[67]$} \\
\hline RCS reduction & GA & $10 \mathrm{GHz}$ & 1 -bit & 2018 & {$[71]$} \\
\hline Wave modulation & GA & $10 \mathrm{GHz}$ & 3 -bit & 2018 & {$[72]$} \\
\hline Harmonic steering & PSO & $10 \mathrm{GHz}$ & $3-$-bit & 2018 & {$[28]$} \\
\hline Multi-beam steering & Addition & $9 \mathrm{GHz}$ & 3 -bit & 2018 & {$[27]$} \\
\hline Asymmetric split & Addition & $10 \mathrm{GHz}$ & 3 -bit & 2019 & {$[68]$} \\
\hline Wavefront shaping & Add./Conv. & $1 \mathrm{THz}$ & 3 -bit & 2019 & {$[73]$} \\
\hline Focusing & Analytical & $2 \mathrm{THz}$ & 2 -bit & 2019 & {$[19]$} \\
\hline Nonreciprocal refl. & Analytical & $10 \mathrm{GHz}$ & 2 -bit & 2019 & {$[57]$} \\
\hline Beamforming & Neural net & $10 \mathrm{GHz}$ & 1 -bit & 2019 & {$[74]$} \\
\hline Beam steering & Analytical & $30 \mathrm{GHz}$ & $2-$-bit & 2019 & {$[75]$} \\
\hline Tunable scattering & Analytical & $1.2 \mathrm{THz}$ & 2 -bit & 2020 & {$[76]$} \\
\hline
\end{tabular}

PSO = Particle Swarm Optimization, GA = Genetic Algorithm, $\mathrm{SA}=$ Simulated Annealing, GS $=$ Gerchberg-Saxton .

in fact, an explosion of works attempting to demonstrate the benefits of programmable metasurfaces and the multiple possibilities they enable across the spectrum. Table I shows a selection of representative designs of the state of the art in coding metasurfaces.

However, the vision of programmable metasurfaces for software-driven EM control goes beyond the achievement of particular EM functionalities and involves groundbreaking as well as, in occasions, much-anticipated non-trivial features. We next list some of such features and relate them with the emerging applications outlined in Section II-A:

- Space-time reconfigurability: as we have seen in Section II-C, metasurfaces have traditionally encoded spatial patterns within their structure in the form of spatial phase or amplitude profiles giving rise to the required functionalities [64]. The advent of programmable metasurfaces, however, introduced the dimension of time [28], [77]: metasurfaces can now be temporally modulated to implement non-reciprocal functionalities [78], compensate for relativistic motion [79], integrate signal and beam modulation within the antenna [38], enable new modulation schemes [80], and many others.

- Multi-functionality: the idea of a metasurface platform that can implement arbitrary spatiotemporal patterns directly leads to think on the implementation of multiple functions or channels within the same metasurface, be it in a discrete/multiplexed or a combined/concurrent fashion [23], [70], [81]. Designs are thus reusable for multiple tasks or a single complex task where the metasurface needs to exhibit different behaviors simultaneously, as in programmable wireless environments [32], [39]. Combined with space-time reconfigurability, this leads to very powerful control of EM waves [77].

- Learnability: the addition of software mechanisms abstracting the physics enables the use of not only optimization techniques, but also machine learning algorithms to model, predict, and control the operation of the 
metasurfaces for arbitrary functionalities and environment conditions. Moreover, with the embedding of intelligence within the metasurface, the metamaterial could automatically adapt to changes in the environment without the intervention of external components.

- Interconnectivity: by providing metamaterials with means of internal and external communication, we are opening the door to the coordination of multiple metamaterials to cover large areas or implement heterogeneous, complex, emerging behaviors. Thanks to multifunctionality, this does not imply the deployment of a set of specialized metasurfaces, but rather the use a single type of metamaterials that changes its behavior according to the role that it is assigned within the swarm. This has obvious implications in the programmable wireless environments paradigm [32], [39].

- Resilience: enabling self-adaptivity of metamaterials to changes in the environment also opens the door, naturally, to changes in the internal structure of the metamaterial due to transient or persistent faults. Although it has been shown that metasurfaces are inherently tolerant to faults of a fraction of unit cells [82], one can envisage designs where the impact can be minimized by incorporating, within the learning/optimization framework, their possible occurrence.

- Perpetual Operation: as metasurfaces become active elements that consume power, a concern is whether the power consumption will impact on the autonomy or applicability of metamaterials. Through the embedding of intelligence and self-adaptive optimization frameworks, however, one can envisage platforms that minimize power consumption by, for instance, keeping only the indispensable features alive. Power-gated unit cells can be considered as faults and resort to the resilience of the platform to still adapt performance to the power restrictions [82]. Furthermore, perpetual operation is a desirable trait arising from the combination of ultralow power consumption and the likely incorporation of energy harvesting means within the platform, as discussed in Sec. VI.

- Terahertz Operation: metamaterial and metasurface designs in the terahertz band already exist [9], although most of them are either theoretical or with limited reconfigurability properties. Given the multiple opportunities arising in the fields of imaging [35] or wireless communications [34], among others, there is an interest to take the programmable metasurface paradigm to the $\mathrm{THz}$ band and beyond. However, this poses important challenges in the technological front, as discussed in Sec. V.

\section{ARChitectures}

The programmability in metasurfaces is enabled by the addition of both tuning elements at the unit cells and appropriate control circuits across the metasurface. The control sub-system, in essence, may implement a control loop that is able to modify the response of the metasurface with a given maximum rate, e.g. a few $\mathrm{kHz}$. Such a speed will be determined by the characteristics of the control sub-system and by how it communicates with the metasurface sub-system, always trying to meet the reconfiguration requirements of the final application.

There are different ways to combine and interconnect the metasurface and control sub-systems (also referred to as

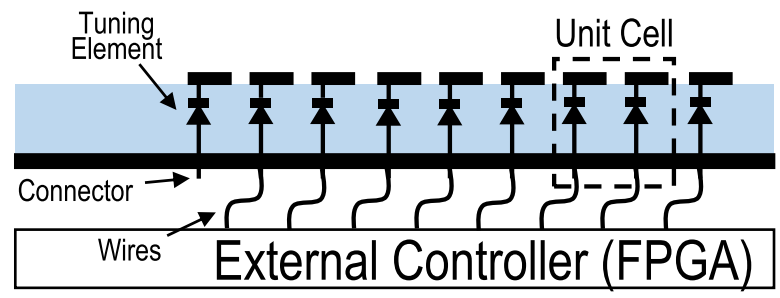

(a) FPGA-based architecture

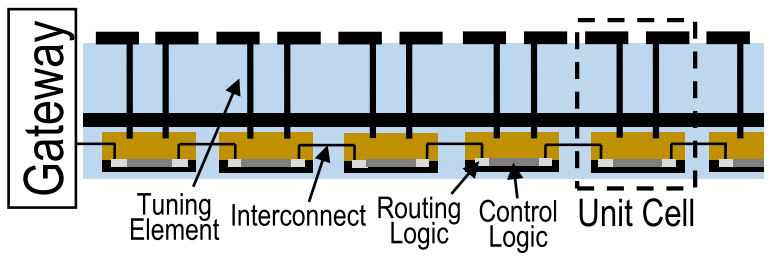

(b) Fully integrated architecture

Fig. 6. Schematic representation of the two general types of programmable metasurface architectures.

physical core and algorithm core), which can be essentially classified into two main architectures. They are illustrated in Figure 6 and described next.

\section{A. FPGA-Based Architectures}

The general FPGA-based architecture of a programmable metasurface system is represented in Figure 6(a) as seen in multiple works [14], [21], [28], [57]. The metasurface itself is responsible for providing programmable electromagnetic characteristics such as reflection/transmission amplitude, phase, polarization, etc. The control sub-system, on the other hand, is mainly composed of an FPGA-based control circuit, which is assembled to implement the functional algorithm and drive the programmable metasurface. The specific configuration of the interface or connector between the two sub-systems depends on the tuning technology used in the metasurface. Several examples include the voltage control circuit for the varactor/PIN diode based metasurface [14], [21], [28], [57], the light intensity control circuit for the photosensitive materials based metasurface [83], and the temperature control circuit for thermo-sensitive materials-based metasurfaces [84], [85].

The separated architecture allows the two sub-systems to be designed independently, which is beneficial to the function expansion and performance upgrade of the entire system through the use of different types of metasurfaces and FPGA models. Therefore, considering cost, difficulty, convenience, etc., numerous proof-of-concept systems have been developed using the FPGA-based architecture [14], [21], [28], [57]. Desired attributes such as space-time multi-functionality, learnability, and interconnectivity are also possible. However, like the other side of a coin, such rough and non-customized architecture is usually bulky and suffers from significant power consumption - hence turning perpetual operation into a complex possibility and, in overall, limiting the applicability and integrability of such a platform in real-world scenarios. Also, the FPGA is a potential single point of failure, rendering the metasurface useless if the FPGA breaks down.

\section{B. Integrated Architectures}

Opposed to the FPGA-based approach, researchers and engineers have started to propose integrated architecture-based 
systems [24], [29], [32], [37]. This paradigm, represented in Figure 6(b) and sometimes referred to the HyperSurface (HSF), indeed hinges on the integration of a network of communicating chips within the metasurface containing tuning elements, control circuits, and even sensors. Attached to the metasurface, a gateway device with wireless connectivity can interface the EM device with the real-world in a cyberphysical/IoT manner. Essentially, the metasurface receives reconfiguration requests that are disseminated to the internal control logic via chip-to-chip interconnects and routing logic [86], [87]. Controllers may consist of simple circuitry that reads the unit cell state and Digital/Analog (D/A) converters that set the appropriate voltage to the tuning elements, or more complex circuits implementing the desired intelligence. Additionally, embedded sensors can pick up data from the environment and send it to the control logic or external devices again via this communications plane.

Integrated architectures are custom-made and therefore are much more optimized than FPGA-based architectures. This means that the control sub-system is clearly less intrusive in terms of EM interference, less bulky, and with lower potential power consumption. For instance, movement of control data among controllers can be measured and predicted, opening the door to streamlined interconnect designs [88]. Furthermore, the addition of an internal network of controllers instead of a centralized FPGA provides metasurfaces with means to implement the so-called distributed intelligence allowing controllers to sense, cooperate, and act locally without the intervention of an external entity [89]. This avoids central-point bottlenecks and improves resilience by allowing information to be rerouted to reach the intended destinations even if a set of connections fail [86].

All these features, however, come at the cost of increased engineering efforts and cost to design and embed the necessary elements within the metasurface. This is especially true as the unit cell density increases with the metasurface frequency, which requires a proportional miniaturization of the control circuitry [29]. Further, the integrated approach lacks the flexibility and convenience of the FPGA-based alternative. More details are given in the next section.

\section{Devices AND Circuits}

From the perspective of devices and circuits, the fully integrated architecture [Fig. 6(b)] presents multiple challenges and considerations related to the tuning devices and methods [11], [12], as well as the efficient integration of such elements and possible interference of control circuits with the operation of the metasurface itself. We discuss a few of these challenges next.

Much has been achieved so far just through the use of the "humble" PIN diode in adaptive metasurfaces [14], [21]. The diode essentially behaves as a capacitor when it is under zero forward bias voltage and it can behave as a resistive element for small signals when forward biased. By carefully combining the diode with a binary DC-voltage driven radial stub and a square patch creates unit cells whose reflection phase can be switched between 0 and $\pi$. Though a simple but very powerful technique, this has room for improvement when it comes to power consumption and fine adaptation; because forwardbiased diodes consume much current. Intermediate reflection phases can be achieved by changing the bias voltage, however having a discrete D/A converter for each unit cell would be both costly and impractical in terms of the space required on the auxiliary Printed Circuit Board (PCB). This leads to the necessity to take advantage of integrated circuit (IC) technology, whereby a single integrated circuit can contain digitally programmable adaptive impedance loading elements and networking communication circuitry. This is especially important if the system is to be scalable so as to cover an arbitrarily large surface.

The requirements for a practical programmable metasurface are quite challenging. In the most basic of forms a programmable metasurface requires a 3-layer PCB where the top layer is used for a patterned conductive array, the middle layer is a ground plane, used to decouple the EM effects of where the metasurface will be installed, and finally the bottom layer is where the active control components will be placed. Space on the bottom layer is extremely limited, especially if independent control of each unit cell is required. Solutions with conventional IC packages are not viable as they are not cost effective, but also the in-package wirebonds tend to introduce significant parasitic inductances and resistances into the system that have large random variations. Thus, wafer-levelchip-scale-packaging (WLCSP) is the only practical option. The size of these components tend to limit the maximum frequency of a system [90], given that, as the frequency goes up, the unit cell pitch becomes smaller.

The custom ASIC alleviates the space problem on the bottom of the PCB, however this comes with other challenges. For instance, on-chip inductors are relatively lossy, as are other key components such as on-chip varactors and varistors. A technology must be selected that balances economic cost with maximum frequency performance. From the digital control side, there are also challenges with regards to the digital inter-chip communication schemes, given pin constraints and the difficulty of producing a global clock on a arbitrarily scalable metasurface that will not be skewed enough to contain problems. Furthermore, clocking a large surface is very energy intensive and, in some applications such as EM absorbers, this is totally unacceptable given that each clock cycle will produce extensive self-EM emissions. Given these challenges, asynchronous digital architectures appear to be the best solution, especially if global unit cell reconfiguration does not occur frequently. A more detailed discussion on these issues can be found in [90].

The future is bringing exciting new passive two terminal devices, which will enable programmable metasurfaces to reach a new level, both in terms of spatial resolution as well as zero power consumption once configured. The Chua Memcomponents, namely Memristor, Memcapacitor and Meminductor will enable the reconfiguration of the unit cells through a crossbar array using pulses generated by a device on the perimeter, and these passive devices will hold their resistance, capacitance and inductance without consuming any power, until another charge packet is sent to the two-terminal device. The concept was first described in 2018 [91], and new developments using conductive filament based-memristors show promise for low power configurations, that can work at very high frequencies, giving tunable resistance, inductance and capacitance in $\mathrm{nm}$-sized passive devices.

\section{TECHNOLOGY}

The concept of programmable metamaterials is relatively recent and, as such, most of existing realizations are rough 
prototypes, proof-of-concept FPGA-based architectures that do not push the limits in terms of the involved technologies. This is because being mostly in the microwave band, unit cells are large enough to fit the required tuning elements and the back-side connectors. Moreover, the metasurface is tested in several steady-state conditions or for functionalities that do not require a high refresh rate, e.g., several tens of $\mathrm{Hz}$ is more than enough for single-beam steering [88]. Under these conditions, programmable metasurfaces could be built using affordable components and relatively old technologies.

However, the quest for fully-integrated intelligent multifunctional metasurfaces with space-time programmability or simply aiming at the mmWave- $\mathrm{THz}$ bands for much-anticipated applications is likely to bring technological challenges at different levels. One important challenge is to scale the fabrication process of programmable metasurfaces to relatively large areas and arbitrary shapes. A possible solution comes from the architecture perspective: one could resort to modular tile designs that leverage interconnectivity to be clipped together in the required application size and shape [90]. This way, widespread fabrication and integration techniques could be employed, reducing costs and enabling scalability.

This and other issues such as the choice of substrate materials [92], the practicality of the tuning elements [11], [12], or the space left to integrate controllers and $\mathrm{I} / \mathrm{O}$ pins at the unit cell level are greatly dependent on the metasurface frequency, whereas the computational requirements of the control logic hinge on the refresh rate imposed by the final applications. Within this context, we next review the prospects of promising technologies towards high-speed high-frequency programmable metasurfaces.

Conventional materials used for tunable/programmable metasurface are conducting oxides (e.g. ITO) [94], [95], phase change materials [96], liquid crystals [97], and approaches that integrate dielectrics with electro-mechanical systems [15]. Recent emergence of a class of atomically thin materials [98], most notably graphene, transition metal dichalcogenides, among many others are most natural materials platform. Their atomic thickness implies that their electronic properties are very amenable to the effect of electric field. For example, the electron surface densities in these materials are highly tunable in a typical back-gated device geometry [99], [100]. These materials also exhibit strong light-matter interactions via polaritonic resonances [101], [102], due to their inherent dipole excitations such as plasmons, phonons or excitons.

In recent years, there has been significant advancement in graphene plasmonics [103] for various terahertz and mid-infrared applications, such as infrared detectors, filters, modulators, biosensing, to general metasurface applications. Plasmons in graphene are excited by patterning graphene into nanostructures [8], [104], and their plasmon resonances reside in the terahertz to mid-infrared and are also tunable electrically. General reflection and amplitude phase modulation of these graphene plasmonic resonators enable very diverse beam manipulation applications [18], [19], [65]. This includes general beam forming [105], cloaking [106], tunable absorption [107], or polarization control [108], among many others. Experimental demonstration of these various concepts are also emerging [109]-[111]. The performance of these devices are mainly limited by the graphene or substrate losses, hence, restricting the reflection phases to less than the ideal $2 \pi$.
The fundamental and limiting plasmon damping pathways in graphene nanostructures are via the graphene intrinsic phonons and scattering from the edges [104]. Much like the case of metallic nanoparticles [112], the inhomogeneity of the ribbon width can lead to plasmon damping due to the atomic edge defects. Although plasmon excitations in most graphene plasmonic devices lie outside of the Landau damping regime, atomic edge defects can provide the required momentum transfer leading to plasmon decay into electronhole pairs. The damping rate goes as $a / W$, where $a$ is of order of the Fermi velocity in graphene, and $W$ is the ribbon width. The intrinsic phonons in graphene and those from the substrate can also strongly influence the plasmon lifetimes when the plasmons are tuned to these phonon frequencies. In fact, a plasmon lifetime of 20 femto-seconds and smaller is observed when damping through the emission of an optical phonon is allowed [104], [113]. The use of hexagonal boron nitride $(\mathrm{hBN})$ as a substrate for graphene has also been demonstrated to significantly improve the plasmon quality factor [114]. However, as with any materials development, quality will continue to improve. Large scale growth of both graphene and hBN has progressed in recent years [115], [116], leading to centimeter scale single crystals.

Another practical technological consideration in 2D materials metasurface design is appropriate photonic engineering to boost light-matter interactions. A homogeneous graphene sheet has a free space optical absorption of less than $1 \%$ in the midinfrared regime [117]. Graphene plasmonic resonators, on the other hand, have resonant absorption of order $10 \%$ in the mid-infrared regime [104]. To achieve total light absorption, a common approach is to include a back metal reflector positioned at quarter wavelength underneath graphene [118], [119]. However, it is not efficient to use the same metal reflector as the back gate for electrostatic doping control of graphene, since the gate capacitance is small. There are various approaches in the literature in circumventing this issue. One approach involves the use of thin metallic layers placed at nanometer scale underneath the graphene layer [120] as the gate electrodes. It was experimentally demonstrated that total light absorption can be achieved in this $2 \mathrm{D}$ materials metasurface design [120].

Fig. 7(a) illustrates a particular design of graphene based metasurface which is theoretically shown to dynamically tune the scattered wavefront over a full $2 \pi$ phase space [93]. The unit cell of the proposed structure is denoted as a metamolecule, which consists of a pair of noble metal antennas each independently contacting a strip of graphene nanoribbon i.e. plasmonic resonator. Electrical biasing between the noble metal antenna and the global back ground plane would then enable the independent tuning of the Fermi level (i.e. $E_{F 1}$ and $E_{F 2}$ ) in each of the graphene nanoribbons within the metamolecule. The pair of Fermi levels thus constitute the two parameters allowing for the modulation of both amplitude and phase of each metamolecule. Using experimentally realistic design parameters like conservative graphene carrier mobility of $1000 \mathrm{~cm}^{2} \mathrm{~V}^{-1} \mathrm{~s}^{-1}$, simulation results show that the two parameters $E_{F 1}$ and $E_{F 2}$ allow one to independently control the phase and amplitude of the reflected beam of an incoming free space $7 \mu \mathrm{m}$ light, as illustrated in Fig. 7(b)-(e). Further, experimental efforts to-date have demonstrated key advancements in integrating high-quality graphene into backgated devices [114], [121], integration of ultra-smooth metallic components utilizing atomic layer deposition achieving strong 

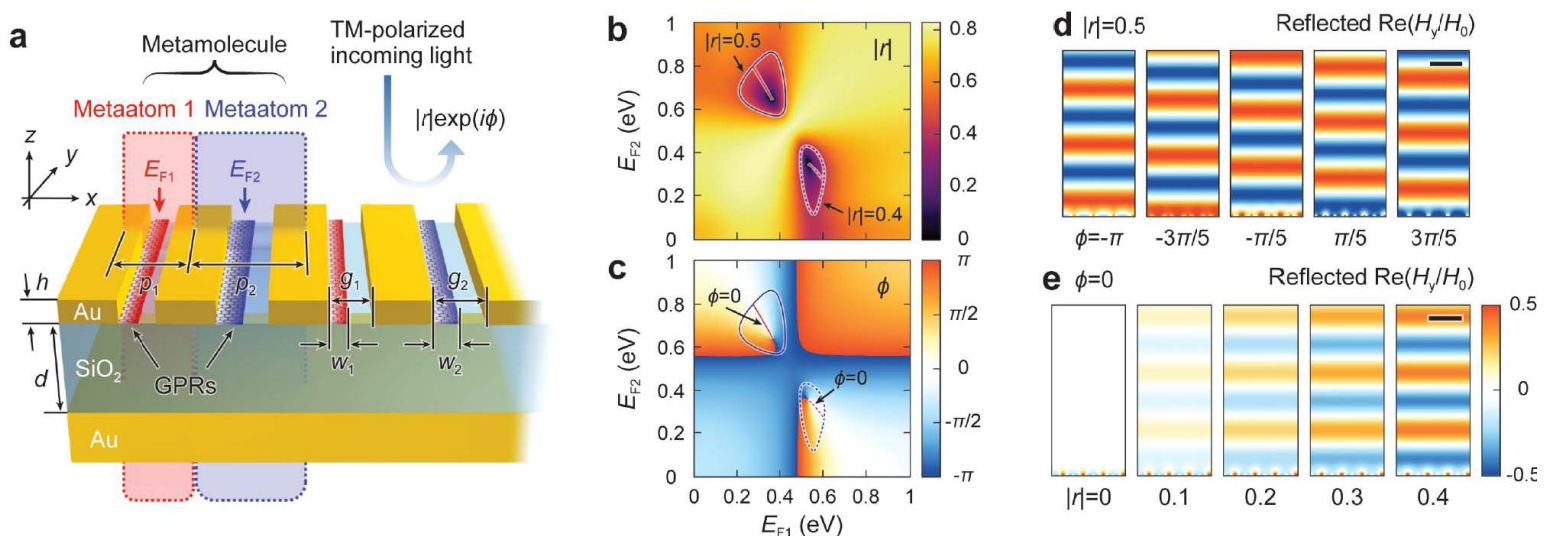

Fig. 7. (a) Schematic of the unit cell structure, denoted as metamolecule, composed of a pair of independently controlled graphene plasmonic metaatoms. (b) Amplitude map and (c) phase map of the reflection as independently adjusting the graphene Fermi levels $\left(E_{F 1}\right.$ and $\left.E_{F 2}\right)$ of the meta-atoms. Reflected transverse magnetic field components $\left(H_{y} / H_{0}\right)$ displaying (d) phase modulation with a constant amplitude $(|r|=0.5)$ along the solid blue line and (e) amplitude modulation including perfect absorption with a constant phase $(\varphi=0)$ along the solid red line. See [93] for more details.

light-matter interaction [120], independent meta-element gate tuning [122], among many others. However, a full experimental demonstration of complete phase and amplitude modulation for efficient beam forming is still in the works.

In summary, graphene, 2D materials, or semiconductor quantum wells [123], present tremendous new device opportunities in the field of terahertz and mid-infrared metasurfaces. However, in order to fully exploit their unique properties or to harness other opportunities such as the use of flexible electronics, it may become necessary to go beyond conventional lithography, etching, or deposition processes. The main hurdle for the manufacturing of $\mathrm{THz}$ metasurfaces is the scaling to surface areas that make sense for the applications at hand. In this direction, the modular approach discussed above is also applicable here. Moreover, besides conventional micromachining methods [9], researchers are pushing towards methods such as transfer printing [124] or deterministic nano-assembly [125]. In the particular case of graphene, wafer-level manufacturing and integration at the graphene qualities required for metasurface applications remains as an outstanding challenge. Many efforts have been devoted recently in this respect [126]-[128] which could allow, in the near future, to electrically gate small graphene patches in relatively large wafers, in the pathway to the much-sought local biasing of graphene -key to achieve the necessary phase modulation in $\mathrm{THz}$ metasurfaces.

\section{Cross-Cutting Issues}

The field of programmable metamaterials is thriving and new research works continue to appear which propose novel cross-cutting approaches with disruptive potential in this emerging field. Next, we briefly describe a few of such aspects and attempt to anticipate its significance.

\section{A. Neural Networks}

Machine learning methods have become all-pervasive in engineering and have been recently proposed for $\mathrm{RF} /$ photonics engineering in general and for the modelling, prediction, and control of metasurfaces in particular. The key benefit of machine learning is that it can simplify tedious design processes like those found in programmable metamaterials, from the unit cell up to the application. First proposals have already appeared proposing to use Convolutional Neural Networks (CNNs) to design broadband unit cells for anisotropic coding metamaterials [129], or Generative Adversarial Networks (GANs) for the inverse design of unit cells with arbitrary spectra [130]-[132]. More recently, interest has been shown in using CNNs to automatically find the most suitable coding pattern for a particular functionality -an approach that has been proven for beam steering [74], but that is also theoretically applicable to arbitrary functionalities. At the application level, machine learning has been also proposed for the control and coordination of multiple metasurfaces in programmable wireless environments [133] or the optimization of metasurface-based imaging in real time [134].

\section{B. Energy Harvesting}

When relating energy harvesting with metamaterials, the main direction has been the use of metamaterials to enable high-efficiency energy harvesting [135]. However, the advent of programmable metasurfaces with low-power integrated architectures has opened a new perspective. Metasurfaces are constantly impinged by EM waves and, theoretically, part of this energy could be absorbed and reused to drive the integrated electronics, as proposed in [29], [136]. This is also encouraged by some wireless communication applications, where users may transmit intermittently in predictable timeslots as defined by the underlying communication protocols; thus opening the door to harvesting the rest of the time. It thus becomes apparent that achieving the objective of perpetual operation may hinge on efforts at the device level (i.e., the energy harvesting device), architecture level (i.e. which energy source to harvest and where/how to place the harvesting devices to that end), and application level (i.e. when to opportunistically harvest).

\section{Fault Tolerance and Approximate Computation}

Recent studies suggest that metasurfaces are naturally resilient to errors in a fraction of the unit cells [82]. In fact, it has been observed that non-trivial phase deviations in the unit cells may be tolerated. This can be exploited in ultralow power designs by operating at low voltages and allowing the system to make transient faults that may be acceptable in certain applications. Leveraging fault tolerance will thus 
impact the design of the controller circuits, as well as the complete architecture in ways that maximize the efficiency benefit while limiting the performance degradation within acceptable bounds.

\section{Internet of Things (IoT)}

Providing programmable metasurfaces with wireless communication capabilities enables the cyber-physical/IoT vision mentioned in the introduction. As such, programmable metamaterials can be introduced in the control or management loop of IoT contexts, enabling unique approaches in a variety of applications, e.g. operators remotely managing wide-area programmable wireless environments.

\section{CONCLUSION}

The field of programmable metamaterials is thriving. Multiple working prototypes have appeared which illustrate the potential of providing metasurfaces with tuning elements and the appropriate control circuitry. Further, a myriad of conceptual works have shown that, with the introduction of modeling strategies such as the coding metamaterial approach or the use of neural networks, the programmable metamaterial paradigm may not only be generalized to virtually any electromagnetic functionality, but also become intelligent and self-adaptive. For this to happen beyond the proof-of-concept stage and in a fully-integrated way, however, advances are required at the technology, circuit, and system levels. Significant challenges will be faced as we push the electromagnetic frequency towards mmWave- $\mathrm{THz}$ bands and as we scale the refresh rate towards $\mathrm{kHz}-\mathrm{MHz}$ magnitudes, both necessary for the realization of compelling applications in the wireless communications or holography fields, among many others.

\section{ACKNOWLEDGMENT}

The authors would like to thank the members of the consortia of their respective projects for the fruitful technical discussions on the matter.

\section{REFERENCES}

[1] J. B. Pendry, "Controlling electromagnetic fields," Science, vol. 312, no. 5781, pp. 1780-1782, Jun. 2006.

[2] S. B. Glybovski, S. A. Tretyakov, P. A. Belov, Y. S. Kivshar, and C. R. Simovski, "Metasurfaces: From microwaves to visible," Phys. Rep., vol. 634, pp. 1-72, May 2016.

[3] A. Li, S. Singh, and D. Sievenpiper, "Metasurfaces and their applications," Nanophotonics, vol. 7, no. 6, pp. 989-1011, Jun. 2018.

[4] S. Vellucci, A. Monti, M. Barbuto, A. Toscano, and F. Bilotti, "Satellite applications of electromagnetic cloaking," IEEE Trans. Antennas Propag., vol. 65, no. 9, pp. 4931-4934, Sep. 2017.

[5] N. I. Landy, S. Sajuyigbe, J. J. Mock, D. R. Smith, and W. J. Padilla, "Perfect metamaterial absorber," Phys. Rev. Lett., vol. 100, no. 20, May 2008, Art. no. 207402.

[6] M. Gokkavas et al., "Experimental demonstration of a left-handed metamaterial operating at $100 \mathrm{GHz}$," Phys. Rev. B, Condens. Matter, vol. 73, no. 19, May 2006, Art. no. 193103.

[7] Z. Zhao et al., "Experimental demonstration of 16-Gbit/s millimeterwave communications link using thin metamaterial plates to generate data-carrying orbital-angular-momentum beams," in Proc. IEEE Int. Conf. Commun. (ICC), Jun. 2015, pp. 1392-1397.

[8] L. Ju et al., "Graphene plasmonics for tunable terahertz metamaterials," Nature Nanotechnol., vol. 6, no. 10, pp. 630-634, 2011.

[9] S.-W. Qu, H. Yi, B. J. Chen, K. B. Ng, and C. H. Chan, "Terahertz reflecting and transmitting metasurfaces," Proc. IEEE, vol. 105, no. 6, pp. 1166-1184, Jun. 2017.
[10] W. Cai and V. M. Shalaev, Optical Metamaterials, vol. 10, no. 6011. New York, NY, USA: Springer-Verlag, 2010.

[11] G. Oliveri, D. H. Werner, and A. Massa, "Reconfigurable electromagnetics through Metamaterials-A review," Proc. IEEE, vol. 103, no. 7, pp. 1034-1056, Jul. 2015.

[12] F. Liu et al., "Programmable metasurfaces: State of the art and prospects," in Proc. IEEE Int. Symp. Circuits Syst. (ISCAS), 2018, pp. $1-5$.

[13] Z. Luo, J. Long, X. Chen, and D. Sievenpiper, "Electrically tunable metasurface absorber based on dissipating behavior of embedded varactors," Appl. Phys. Lett., vol. 109, no. 7, Aug. 2016, Art. no. 071107.

[14] H. Yang et al., "A programmable metasurface with dynamic polarization, scattering and focusing control," Sci. Rep., vol. 6, no. 1, Oct. 2016, Art. no. 35692.

[15] E. Arbabi, A. Arbabi, S. M. Kamali, Y. Horie, M. Faraji-Dana, and A. Faraon, "MEMS-tunable dielectric metasurface lens," Nature Commun., vol. 9, no. 1, 2018, Art. no. 812.

[16] M. Manjappa et al., "Reconfigurable MEMS fano metasurfaces with multiple-input-output states for logic operations at terahertz frequencies," Nature Commun., vol. 9, no. 1, p. 4056, Oct. 2018.

[17] S. Liu, L. Zhang, G. D. Bai, and T. J. Cui, "Flexible controls of broadband electromagnetic wavefronts with a mechanically programmable metamaterial," Sci. Rep., vol. 9, no. 1, Feb. 2019, Art. no. 1809.

[18] S. R. Biswas et al., "Tunable graphene metasurface reflectarray for cloaking, illusion, and focusing," Phys. Rev. Appl., vol. 9, no. 3, Mar. 2018, Art. no. 034021

[19] S. E. Hosseininejad et al., "Reprogrammable graphene-based metasurface mirror with adaptive focal point for THz imaging," Sci. Rep., vol. 9, no. 1, Feb. 2019, Art. no. 2868.

[20] S. Savo, D. Shrekenhamer, and W. J. Padilla, "Liquid crystal metamaterial absorber spatial light modulator for THz applications," Adv. Opt. Mater, vol. 2, no. 3, pp. 275-279, Jan. 2014.

[21] T. J. Cui, M. Q. Qi, X. Wan, J. Zhao, and Q. Cheng, "Coding metamaterials, digital metamaterials and programmable metamaterials," Light, Sci. Appl., vol. 3, no. 10, p. e218, Oct. 2014.

[22] L. Li et al., "Electromagnetic reprogrammable coding-metasurface holograms," Nature Commun., vol. 8, no. 1, pp. 1-7, Aug. 2017.

[23] F. Liu et al., "Intelligent metasurfaces with continuously tunable local surface impedance for multiple reconfigurable functions," Phys. Rev. Appl., vol. 11, no. 4, Apr. 2019, Art. no. 044024.

[24] A. Pitilakis et al., "Software-defined metasurface paradigm: Concept, challenges, prospects," in Proc. 12th Int. Congr. Artif. Mater. Novel Wave Phenomena (Metamaterials), Aug. 2018, pp. 483-485.

[25] T.-J. Cui, S. Liu, and L.-L. Li, "Information entropy of coding metasurface," Light, Sci. Appl., vol. 5, no. 11, p. e16172, Jun. 2016.

[26] S. Liu et al., "Convolution operations on coding metasurface to reach flexible and continuous controls of terahertz beams," Adv. Sci., vol. 3, no. 10, Jul. 2016, Art. no. 1600156.

[27] R. Y. Wu, C. B. Shi, S. Liu, W. Wu, and T. J. Cui, "Addition theorem for digital coding metamaterials," Adv. Opt. Mater, vol. 6, no. 5, Jan. 2018, Art. no. 1701236.

[28] L. Zhang et al., "Space-time-coding digital metasurfaces," Nature Commun., vol. 9, no. 1, p. 4334, 2018.

[29] S. Abadal et al., "Computing and communications for the softwaredefined metamaterial paradigm: A context analysis," IEEE Access, vol. 5, pp. 6225-6235, 2017.

[30] Q. Ma, G. D. Bai, H. B. Jing, C. Yang, L. Li, and T. J. Cui, "Smart metasurface with self-adaptively reprogrammable functions," Light, Sci. Appl., vol. 8, no. 1, pp. 1-12, Oct. 2019.

[31] S. Hu, F. Rusek, and O. Edfors, "Beyond massive MIMO: The potential of data transmission with large intelligent surfaces," IEEE Trans. Signal Process., vol. 66, no. 10, pp. 2746-2758, May 2018.

[32] C. Liaskos, S. Nie, A. Tsioliaridou, A. Pitsillides, S. Ioannidis, and I. Akyildiz, "A new wireless communication paradigm through software-controlled metasurfaces," IEEE Commun. Mag., vol. 56, no. 9, pp. 162-169, Sep. 2018

[33] O. Abari, D. Bharadia, A. Duffield, and D. Katabi, "Cutting the cord in virtual reality," in Proc. 15th ACM Workshop Hot Topics Netw. (HotNets), 2016, pp. 162-168.

[34] I. F. Akyildiz, J. M. Jornet, and C. Han, "Terahertz band: Next frontier for wireless communications," Phys. Commun., vol. 12, pp. 16-32, Sep. 2014.

[35] D. M. Mittleman, "Twenty years of terahertz imaging [Invited]," Opt. Express, vol. 26, no. 8, pp. 9417-9431, Apr. 2018. 
[36] S. Liu and T. J. Cui, "Concepts, working principles, and applications of coding and programmable metamaterials," Adv. Opt. Mater., vol. 5 , no. 22, 2017, Art. no. 1700624

[37] C. Liaskos, S. Nie, A. Tsioliaridou, A. Pitsillides, S. Ioannidis, and I. Akyildiz, "Realizing wireless communication through softwaredefined HyperSurface environments," in Proc. IEEE 19th Int. Symp. World Wireless, Mobile Multimedia Networks (WoWMoM), Jun. 2018, pp. $14-15$.

[38] J. Zhao et al., "Programmable time-domain digital-coding metasurface for non-linear harmonic manipulation and new wireless communication systems," Nat. Sci. Rev., vol. 6, no. 2, pp. 231-238, Nov. 2018.

[39] C. Liaskos, A. Tsioliaridou, A. Pitsillides, S. Ioannidis, and I. Akyildiz, "Using any surface to realize a new paradigm for wireless communications," Commun. ACM, vol. 61, no. 11, pp. 30-33, Oct. 2018.

[40] Y. Han, W. Tang, S. Jin, C.-K. Wen, and X. Ma, "Large intelligent surface-assisted wireless communication exploiting statistical CSI," IEEE Trans. Veh. Technol., vol. 68, no. 8, pp. 8238-8242, Aug. 2019.

[41] M. D. Renzo et al., "Smart radio environments empowered by reconfigurable AI meta-surfaces: An idea whose time has come," EURASIP J. Wireless Commun. Netw., vol. 2019, no. 1, p. 129, May 2019.

[42] I. F. Akyildiz, C. Han, and S. Nie, "Combating the distance problem in the millimeter wave and terahertz frequency bands," IEEE Commun. Mag., vol. 56, no. 6, pp. 102-108, Jun. 2018.

[43] K. Ntontin et al., "Reconfigurable intelligent surfaces vs. Relaying: Differences, similarities, and performance comparison," 2019 , arXiv:1908.08747. [Online]. Available: http://arxiv.org/abs/1908.08747

[44] J. Y. Dai et al., "Wireless communications through a simplified architecture based on time-domain digital coding metasurface," Adv Mater. Technol., vol. 4, no. 7, Feb. 2019, Art. no. 1900044.

[45] A. Babakhani, D. B. Rutledge, and A. Hajimiri, "Transmitter architectures based on near-field direct antenna modulation," IEEE J. SolidState Circuits, vol. 43, no. 12, pp. 2674-2692, Dec. 2008.

[46] N. Ashraf, T. Saeed, R. I. Ansari, M. Lestas, C. Liaskos, and A. Pitsillides, "Feedback based beam steering for intelligent metasurfaces," in Proc. 2nd IEEE Middle East North Afr. Commun. Conf. (MENACOMM), Nov. 2019, pp. 1-6.

[47] L. Zhang et al., "Transmission-Reflection-Integrated multifunctional coding metasurface for full-space controls of electromagnetic waves," Adv. Funct. Mater., vol. 28, no. 33, Jun. 2018, Art. no. 1802205.

[48] R. Y. Wu et al., "Digital metasurface with phase code and reflectiontransmission amplitude code for flexible full-space electromagnetic manipulations," Adv. Opt. Mater., vol. 7, no. 8, 2019, Art. no. 1801429.

[49] X. Wan et al., "Multichannel direct transmissions of near-field information," Light, Sci. Appl., vol. 8, no. 1, pp. 1-8, Jul. 2019.

[50] K. Chen et al., "A reconfigurable active Huygens' metalens," Adv. Mater., vol. 29, no. 17, Feb. 2017, Art. no. 1606422

[51] L. Liang et al., "Anomalous terahertz reflection and scattering by flexible and conformal coding metamaterials," Adv. Opt. Mater., vol. 3, no. 10, p. 1311, Jul. 2015

[52] K. Chen, Y. Feng, L. Cui, J. Zhao, T. Jiang, and B. Zhu, "Dynamic control of asymmetric electromagnetic wave transmission by active chiral metamaterial," Sci. Rep., vol. 7, no. 1, Feb. 2017, Art. no. 42802.

[53] X. Gao, W. L. Yang, H. F. Ma, Q. Cheng, X. H. Yu, and T. J. Cui, "A reconfigurable broadband polarization converter based on an active metasurface," IEEE Trans. Antennas Propag., vol. 66, no. 11, pp. 6086-6095, Nov. 2018.

[54] L. Zhang, S. Liu, L. Li, and T. J. Cui, "Spin-controlled multiple pencil beams and vortex beams with different polarizations generated by pancharatnam-berry coding metasurfaces," ACS Appl. Mater. Interfaces, vol. 9, no. 41, pp. 36447-36455, Oct. 2017.

[55] J. Zhao, Q. Cheng, J. Chen, M. Q. Qi, W. X. Jiang, and T. J. Cui, "A tunable metamaterial absorber using varactor diodes," New J. Phys., vol. 15, no. 4, Apr. 2013, Art. no. 043049

[56] D. S. Dong et al., "Terahertz broadband low-reflection metasurface by controlling phase distributions," Adv. Opt. Mater., vol. 3, no. 10, pp. 1405-1410, Jun. 2015.

[57] L. Zhang et al., "Breaking reciprocity with space-time-coding digital metasurfaces," Adv. Mater., vol. 31, no. 41, 2019, Art. no. 1904069.

[58] Q. Ma et al., "Controllable and programmable nonreciprocity based on detachable digital coding metasurface," Adv. Opt. Mater., vol. 7, no. 24, Oct. 2019, Art. no. 1901285.

[59] Y. B. Li et al., "Transmission-type 2-Bit programmable metasurface for single-sensor and single-frequency microwave imaging," Sci. Rep., vol. 6, no. 1, pp. 1-8, Mar. 2016.
[60] T. J. Cui, R. Y. Wu, W. Wu, C. B. Shi, and Y. B. Li, "Large-scale transmission-type multifunctional anisotropic coding metasurfaces in millimeter-wave frequencies," J. Phys. D, Appl. Phys., vol. 50, no. 40, Sep. 2017, Art. no. 404002.

[61] S. Liu et al., "Anisotropic coding metamaterials and their powerful manipulation of differently polarized terahertz waves," Light, Sci. Appl., vol. 5, no. 5, p. e16076, Jan. 2016.

[62] G. D. Bai et al., "Multitasking shared aperture enabled with multiband digital coding metasurface," Adv. Opt. Mater, vol. 6, no. 21, Aug. 2018 , Art. no. 1800657.

[63] J. Luo et al., "2-bit amplitude-modulated coding metasurfaces based on indium tin oxide films," J. Appl. Phys., vol. 126, no. 11, Sep. 2019 Art. no. 113102

[64] N. Yu et al., "Light propagation with phase discontinuities: Generalized laws of reflection and refraction," Science, vol. 334, no. 6054, pp. 333-337, Sep. 2011

[65] S. E. Hosseininejad, K. Rouhi, M. Neshat, A. Cabellos-Aparicio, S. Abadal, and E. Alarcon, "Digital metasurface based on graphene: An application to beam steering in terahertz plasmonic antennas," IEEE Trans. Nanotechnol., vol. 18, pp. 734-746, 2019.

[66] K. Wang, J. Zhao, Q. Cheng, D. S. Dong, and T. J. Cui, "Broadband and broad-angle low-scattering metasurface based on hybrid optimization algorithm," Sci. Rep., vol. 4, no. 1, Aug. 2014, Art. no. 5935.

[67] L. R. Ji-Di, X. Y. Cao, Y. Tang, S. M. Wang, Y. Zhao, and X. W. Zhu, "A new coding metasurface for wideband RCS reduction," Radioengineering, vol. 27, no. 2, pp. 394-401, Jun. 2018.

[68] H. Rajabalipanah, A. Abdolali, J. Shabanpour, A. Momeni, and A. Cheldavi, "Asymmetric spatial power dividers using phaseamplitude metasurfaces driven by Huygens principle," ACS Omega, vol. 4, no. 10, pp. 14340-14352, Aug. 2019.

[69] H. Yi, S.-W. Qu, B.-J. Chen, X. Bai, K. B. Ng, and C. H. Chan, "Flat terahertz reflective focusing metasurface with scanning ability," Sci. Rep., vol. 7, no. 1, pp. 2-9, Jun. 2017.

[70] S. Liu et al., "Full-state controls of terahertz waves using tensor coding metasurfaces," ACS Appl. Mater. Interfaces, vol. 9, no. 25, pp. 21503-21514, Jun. 2017.

[71] S. Sui et al., "Absorptive coding metasurface for further radar cross section reduction," J. Phys. D, Appl. Phys., vol. 51, no. 6, Jan. 2018, Art. no. 065603

[72] Y. Zhou et al., "Design of phase gradient coding metasurfaces for broadband wave modulating," Sci. Rep., vol. 8, no. 1, Jun. 2018, Art. no. 8672 .

[73] K. Rouhi, H. Rajabalipanah, and A. Abdolali, "Multi-bit graphenebased bias-encoded metasurfaces for real-time terahertz wavefron shaping: From controllable orbital angular momentum generation toward arbitrary beam tailoring," Carbon, vol. 149, pp. 125-138, Aug. 2019.

[74] T. Shan and M. Li, "Coding programmable metasurfaces based on deep learning techniques," in Proc. AP-S/URSI, 2019.

[75] Q. Wang et al., "Millimeter-wave digital coding metasurfaces based on nematic liquid crystals," Adv. Theory Simulations, vol. 2, no. 12, 2019, Art. no. 1900141.

[76] J. Li et al., "Frequency-switchable $\mathrm{VO}_{2}$-based coding metasurfaces at the terahertz band," Opt. Commun., vol. 458, 2020, Art. no. 124744.

[77] X. Wang, A. Diaz-Rubio, H. Li, S. A. Tretyakov, and A. Alu, "Multifunctional space-time metasurfaces," 2019, arXiv:1910.11812. [Online]. Available: http://arxiv.org/abs/1910.11812

[78] A. Shaltout, A. Kildishev, and V. Shalaev, "Time-varying metasurfaces and Lorentz non-reciprocity," Opt. Mater. Express, vol. 5, no. 11, pp. 2459-2467, Oct. 2015.

[79] D. Ramaccia, D. L. Sounas, A. Alu, A. Toscano, and F. Bilotti, "Phase-induced frequency conversion and Doppler effect with timemodulated metasurfaces," IEEE Trans. Antennas Propag., to be published.

[80] E. Basar, "Reconfigurable intelligent surface-based index modulation: A new beyond MIMO paradigm for 6G," IEEE Trans. Commun., to be published.

[81] V. S. Asadchy et al., "Flat engineered multichannel reflectors," Phys. Rev. X, vol. 7, no. 3, Sep. 2017, Art. no. 031046.

[82] H. Taghvaee, S. Abadal, J. Georgiou, A. Cabellos-Aparicio, and E. Alarcon, "Fault tolerance in programmable metasurfaces: The beam steering case," in Proc. IEEE Int. Symp. Circuits Syst. (ISCAS), May 2019, pp. 1-5.

[83] K. M. Kossifos, M. A. Antoniades, J. Georgiou, A. H. Jaafar, and N. T. Kemp, "An optically-programmable absorbing metasurface," in Proc. IEEE Int. Symp. Circuits Syst. (ISCAS), May 2018, pp. 1-5. 
[84] D. Wang et al., "Multiband switchable terahertz quarter-wave plates via phase-change metasurfaces," IEEE Photon. J., vol. 8, no. 1, Feb. 2016, Art. no. 5500308.

[85] K. Sun et al., "VO $\mathrm{VO}_{2}$ thermochromic metamaterial-based smart optical solar reflector," ACS Photon., vol. 5, no. 6, pp. 2280-2286, 2018.

[86] D. Kouzapas et al., "Towards fault adaptive routing in metasurface controller networks," J. Syst. Archit., vol. 106, Jun. 2020, Art. no. 101703.

[87] A. C. Tasolamprou et al., "Exploration of intercell wireless millimeterwave communication in the landscape of intelligent metasurfaces," IEEE Access, vol. 7, pp. 122931-122948, 2019.

[88] T. Saeed et al., "Workload characterization of programmable metasurfaces," in Proc. the 6th Annu. ACM Int. Conf. Nanosc. Comput. Commun. NANOCOM, 2019, pp. 1-6.

[89] C. Liaskos et al., "ABSense: Sensing electromagnetic waves on metasurfaces via ambient compilation of full absorption," in Proc. 6th Annu. ACM Int. Conf. Nanosc. Comput. Commun. NANOCOM, 2019, pp. 1-6.

[90] L. Petrou, P. Karousios, and J. Georgiou, "Asynchronous circuits as an enabler of scalable and programmable metasurfaces," in Proc. IEEE Int. Symp. Circuits Syst. (ISCAS), May 2018.

[91] J. Georgiou, K. M. Kossifos, M. A. Antoniades, A. H. Jaafar, and N. T. Kemp, "Chua mem-components for adaptive RF metamaterials," in Proc. IEEE Int. Symp. Circuits Syst. (ISCAS), May 2018, pp. 1-5.

[92] D. Manessis et al., "High frequency substrate technologies for the realisation of software programmable metasurfaces on PCB hardware platforms with integrated controller nodes," in Proc. 22nd Eur. Microelectron. Packag. Conf. Exhibit. (EMPC), Sep. 2019, pp. 1-7.

[93] S. Han, S. Kim, S. Kim, T. Low, V. W. Brar, and M. S. Jang, "Complete complex amplitude modulation with electronically tunable graphene plasmonic metamolecules," ACS Nano, vol. 14, no. 1, pp. 1166-1175, Jan. 2020.

[94] Y.-W. Huang et al., "Gate-tunable conducting oxide metasurfaces," Nano Lett., vol. 16, no. 9, pp. 5319-5325, Sep. 2016.

[95] G. Kafaie Shirmanesh, R. Sokhoyan, P. Chieh Wu, and H. A. Atwater, "Electro-optically tunable universal metasurfaces," 2019, arXiv:1910.02069. [Online]. Available: http://arxiv.org/abs/1910. 02069

[96] K. Chaudhary et al., "Polariton nanophotonics using phase-change materials," Nature Commun., vol. 10, no. 1, pp. 1-6, Oct. 2019.

[97] J. Lee et al., "Ultrafast electrically tunable polaritonic metasurfaces," Adv. Opt. Mater., vol. 2, no. 11, pp. 1057-1063, Jul. 2014.

[98] P. Avouris, T. F. Heinz, and T. Low, 2D Materials. Cambridge, U.K.: Cambridge Univ. Press, 2017.

[99] K. S. Novoselov, "Electric field effect in atomically thin carbon films," Science, vol. 306, no. 5696, pp. 666-669, Oct. 2004.

[100] Q. H. Wang, K. Kalantar-Zadeh, A. Kis, J. N. Coleman, and M. S. Strano, "Electronics and optoelectronics of two-dimensional transition metal dichalcogenides," Nature Nanotechnol., vol. 7, no. 11, pp. 699-712, Nov. 2012.

[101] T. Low et al., "Polaritons in layered two-dimensional materials," Nature Mater., vol. 16, no. 2, pp. 182-194, Nov. 2016.

[102] D. N. Basov, M. M. Fogler, and F. J. Garcia de Abajo, "Polaritons in van der Waals materials," Science, vol. 354, no. 6309, p. aag1992, Oct. 2016.

[103] T. Low and P. Avouris, "Graphene plasmonics for terahertz to midinfrared applications," ACS Nano, vol. 8, no. 2, pp. 1086-1101, Jan. 2014.

[104] H. Yan et al., "Damping pathways of mid-infrared plasmons in graphene nanostructures," Nature Photon., vol. 7, no. 5, pp. 394-399, Apr. 2013

[105] A. Fallahi and J. Perruisseau-Carrier, "Design of tunable biperiodic graphene metasurfaces," Phys. Rev. B, Condens. Matter, vol. 86, no. 19, Nov. 2012, Art. no. 195408

[106] P.-Y. Chen, J. Soric, Y. R. Padooru, H. M. Bernety, A. B. Yakovlev, and A. Alù, "Nanostructured graphene metasurface for tunable terahertz cloaking," New J. Phys., vol. 15, no. 12, Dec. 2013, Art. no. 123029.

[107] X. Wang and S. A. Tretyakov, "Toward ultimate control of terahertz wave absorption in graphene," IEEE Trans. Antennas Propag., vol. 67, no. 4, pp. 2452-2461, Apr. 2019.

[108] T. Guo and C. Argyropoulos, "Broadband polarizers based on graphene metasurfaces," Opt. Lett., vol. 41, no. 23, pp. 5592-5595, Nov. 2016.

[109] M. C. Sherrott et al., "Experimental demonstration of $>230$ phase modulation in gate-tunable graphene-gold reconfigurable midinfrared metasurfaces," Nano Lett., vol. 17, no. 5, pp. 3027-3034, Apr. 2017.
[110] N. Dabidian et al., "Experimental demonstration of phase modulation and motion sensing using graphene-integrated metasurfaces," Nano Lett., vol. 16, no. 6, pp. 3607-3615, May 2016.

[111] Z. Miao et al., "Widely tunable terahertz phase modulation with gate-controlled graphene metasurfaces," Phys. Rev. X, vol. 5, no. 4, Nov. 2015, Art. no. 041027

[112] U. Kreibig and M. Vollmer, Optical Properties of Metal Clusters, vol. 25. Berlin, Germany: Springer-Verlag, 2013.

[113] G. X. Ni et al., "Fundamental limits to graphene plasmonics," Nature, vol. 557, no. 7706, pp. 530-533, May 2018.

[114] A. Woessner et al., "Highly confined low-loss plasmons in grapheneboron nitride heterostructures," Nature Mater., vol. 14, no. 4, pp. 421-425, Dec. 2014.

[115] Y. Hao et al., "The role of surface oxygen in the growth of large singlecrystal graphene on copper," Science, vol. 342, no. 6159, pp. 720-723, Oct. 2013.

[116] L. Wang et al., "Epitaxial growth of a 100-square-centimetre singlecrystal hexagonal boron nitride monolayer on copper," Nature, vol. 570, no. 7759, pp. 91-95, May 2019.

[117] Z. Q. Li et al., "Dirac charge dynamics in graphene by infrared spectroscopy," Nature Phys., vol. 4, no. 7, pp. 532-535, Jun. 2008.

[118] E. Carrasco, M. Tamagnone, J. R. Mosig, T. Low, and J. Perruisseau-Carrier, "Gate-controlled mid-infrared light bending with aperiodic graphene nanoribbons array," Nanotechnology, vol. 26, no. 13, Mar. 2015, Art. no. 134002.

[119] M. S. Jang et al., "Tunable large resonant absorption in a midinfrared graphene Salisbury screen," Phys. Rev. B, vol. 90, no. 16, Oct. 2014, Art. no. 165409.

[120] I.-H. Lee, D. Yoo, P. Avouris, T. Low, and S.-H. Oh, "Graphene acoustic plasmon resonator for ultrasensitive infrared spectroscopy," Nature Nanotechnol., vol. 14, no. 4, pp. 313-319, Feb. 2019.

[121] S. S. Sunku et al., "Photonic crystals for nano-light in moiré graphene superlattices," Science, vol. 362, no. 6419, pp. 1153-1156, Dec. 2018.

[122] M. Tamagnone et al., "Graphene reflectarray metasurface for terahertz beam steering and phase modulation," 2018, arXiv:1806.02202. [Online]. Available: http://arxiv.org/abs/1806.02202

[123] P. C. Wu et al., "Dynamic beam steering with all-dielectric electro-optic III-V multiple-quantum-well metasurfaces," Nature Commun., vol. 10 , no. 1, p. 3654, Aug. 2019.

[124] S. Lee et al., "Heterogeneously assembled metamaterials and metadevices via 3D modular transfer printing," Sci. Rep., vol. 6, no. 1, Jun. 2016, Art. no. 27621.

[125] B. Kim et al., "Deterministic nanoassembly of quasi-three-dimensional plasmonic nanoarrays with arbitrary substrate materials and structures," Nano Lett., vol. 19, no. 8, pp. 5796-5805, Jul. 2019.

[126] Y. Lee et al., "Wafer-scale synthesis and transfer of graphene films," Nano Lett., vol. 10, no. 2, pp. 490-493, Feb. 2010.

[127] J. S. Lee et al., "Wafer-scale single-crystal hexagonal boron nitride film via self-collimated grain formation," Science, vol. 362, no. 6416, pp. 817-821, Nov. 2018.

[128] D. Neumaier, S. Pindl, and M. C. Lemme, "Integrating graphene into semiconductor fabrication lines," Nature Mater, vol. 18, no. 6 , pp. 525-529, May 2019.

[129] Q. Zhang et al., "Machine-learning designs of anisotropic digital coding metasurfaces," Adv. Theory Simul., vol. 2, no. 2, Nov. 2018, Art. no. 1800132.

[130] Z. Liu, D. Zhu, S. P. Rodrigues, K.-T. Lee, and W. Cai, "Generative model for the inverse design of metasurfaces," Nano Lett., vol. 18, no. 10, pp. 6570-6576, Sep. 2018.

[131] J. Jiang and J. A. Fan, "Simulator-based training of generative neural networks for the inverse design of metasurfaces," Nanophotonics, Nov. 2019. [Online]. Available: https://www.degruyter.com/view/j/ nanoph.ahead-of-print/nanoph-2019-0330/nanoph-2019-0330.xml? lang=en, doi: 10.1515/nanoph-2019-0330.

[132] W. Ma, F. Cheng, Y. Xu, Q. Wen, and Y. Liu, "Probabilistic representation and inverse design of metamaterials based on a deep generative model with semi-supervised learning strategy," Adv. Mater, vol. 31, no. 35, Jul. 2019, Art. no. 1901111.

[133] C. Liaskos, A. Tsioliaridou, S. Nie, A. Pitsillides, S. Ioannidis, and I. Akyildiz, "An interpretable neural network for configuring programmable wireless environments," in Proc. IEEE 20th Int. Workshop Signal Process. Adv. Wireless Commun. (SPAWC), Jul. 2019, pp. 1-5.

[134] L. Li et al., "Machine-learning reprogrammable metasurface imager," Nature Commun., vol. 10, no. 1, pp. 1-8, Mar. 2019. 
[135] Z. Chen, B. Guo, Y. Yang, and C. Cheng, "Metamaterials-based enhanced energy harvesting: A review," Phys. B, Condens. Matter, vol. 438, pp. 1-8, Apr. 2014.

[136] F. Lemic, R. U. Akbar, J. Marquez-Barja, and J. Famaey, “Assessing the reliability of energy harvesting terahertz nanonetworks for controlling software-defined metamaterials," in Proc. 6th Annu. ACM Int. Conf. Nanosc. Comput. Commun. (NANOCOM), 2019, pp. 1-6.

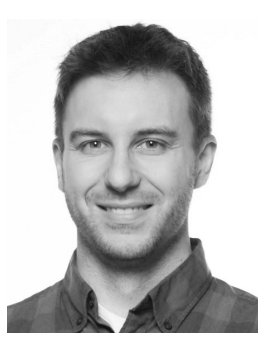

Sergi Abadal (Member, IEEE) received the B.Sc. degree in telecommunication engineering from the Telecommunication Engineering School (Telecom $\mathrm{BCN}$ ), Universitat Politècnica de Catalunya (UPC), Barcelona, Spain, in 2010, the M.Sc. degree in information and communication technologies (MINT) from UPC in 2011, and the Ph.D. degree in computer architecture from the Department of Computer Architecture, UPC, in July 2016. He has held several visiting positions at Georgia Tech in 2009 the University of Illinois at Urbana-Champaign from 2015 to 2016, and the Foundation of Research and Technology - Hellas in 2018. He is currently a Coordinator of the WIPLASH FET-OPEN Project. His current research interests are in the areas of chip-scale wireless communications, including channel modeling and protocol design, the application of these techniques for the creation of next-generation wireless networks for on-chip or within metamaterials, and their implications at the system design level. He has served as a TPC member for more than ten conferences and has organized three special sessions, and published over 60 articles in top-tier journals and conferences. He was a recipient of the Nano Communication Networks Young Researcher Award in 2019, the INTEL Doctoral Fellowship in 2013, the Accenture Award for M.Sc. students in 2012, and the Vodafone Fellowship for B.Sc. students in 2010. He is also an Area Editor of the Nano Communication Networks (Elsevier), where he was named "Editor of the Year" in 2019.

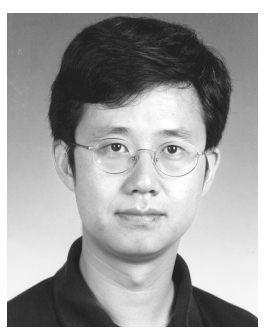

Tie-Jun Cui (Fellow, IEEE) received the B.Sc., M.Sc., and Ph.D. degrees in electrical engineering from Xidian University, Xi'an, China, in 1987, 1990, and 1993 , respectively.

In March 1993, he joined the Department of Electromagnetic Engineering, Xidian University, where he was promoted to an Associate Professor in November 1993. From 1995 to 1997, he was a Research Fellow with the Institut für Hochfrequenztechnik und Elektronik (IHE), University of Karlsruhe, Germany. In July 1997, he joined the Center for Computational Electromagnetics, Department of Electrical and Computer Engineering, University of Illinois at Urbana-Champaign, first as a Post-Doctoral Research Associate and then a Research Scientist. In September 2001, he was a Cheung-Kong Professor with the Department of Radio Engineering, Southeast University, Nanjing, China, where he became a Chief Professor in January 2018. He is the first author of the books Metamaterials - Theory, Design, and Applications (Springer, November 2009) and Metamaterials: Beyond Crystals, Noncrystals, and Quasicrystals (CRC Press, March 2016). He has published over 400 peer-review journal articles in Science, Proceedings of the National Academy of Sciences (PNAS), Nature Communications, Physical Review Letters, Advanced Materials, and the IEEE TRANSACTIONS, which have been cited more than 20000 times (H-Factor 73). According to Elsevier, he is one of the Most Cited Chinese Researchers.

Dr. Cui received the Research Fellowship from the Alexander von Humboldt Foundation, Bonn, Germany, in 1995, the Young Scientist Award from the International Union of Radio Science (URSI) in 1999, the Cheung Kong Professorship under the Cheung Kong Scholar Program by the Ministry of Education, China, in 2001, the National Science Foundation of China for Distinguished Young Scholars in 2002, the Special Government Allowance from the Department of State, China, in 2008, the Award of Science and Technology Progress from the Shaanxi Province Government in 2009, and the May 1st Labour Medal from Jiangsu Province Government in 2010. He also received the First Prize of Natural Science Awards from Ministry of Education, China, in 2011, the Second Prize of National Natural Science Awards, China, in 2014, and the First Prize of Military Science and Technology Progress Awards in 2016. His researches have been selected as one of the "Optics in 2016" by Optics and Photonics News Magazine (OSA), "10 Breakthroughs of China Optics in 2016," "10 Breakthroughs of China Science in 2010," "Best of 2010" in New Journal of Physics, Research Highlights in Europhysics News, Journal of Physics D: Applied Physics, Applied Physics Letters, and Nature China.
$\mathrm{He}$ is an Active Reviewer for Science, Nature Materials, Nature Photonics, Nature Physics, Nature Communications, Physical Review Letters, Advanced Materials, and a series of the IEEE TRANSACTIONS. He was an Associate Editor of the IEEE TRANSACTIONS ON GeOSCIENCE AND REMOTE SENSING and a Guest Editor of Science China - Information Sciences. He served as on the editorial staff for the IEEE Antennas and Propagation Magazine, and is on the Editorial Boards of Progress in Electromagnetics Research (PIER) and Journal of Electromagnetic Waves and Applications.

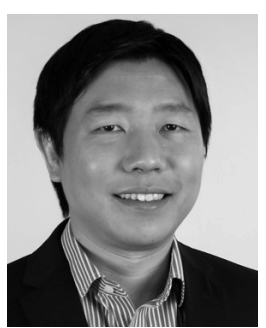

Tony Low received the B.Sc. and Ph.D. degrees in electrical and computer engineering from the National University of Singapore in 2002 and 2008, respectively. After that, he was a Post-Doctoral Associate with Purdue University until 2011, and then, he worked as a Theorist with various experimental groups at Columbia University, Yale University, and IBM Thomas J. Watson Research. While at IBM, from 2011 to 2014, he served as an industry liaison to various universities under the Nanoelectronics Research Initiative with the goal of finding the next electronics switch. He is currently an Associate Professor at the Department of Electrical and Computer Engineering, University of Minnesota. He was also a member of the technical program committees of over ten conferences, given more than 100 invited talks, and published over 100 articles. He has been included in the 2019 Global List of Highly Cited Researchers, compiled by the Web of Science Group. He received the Presidential McKnight Fellowship Award in 2019, the IBM Pat Goldberg Memorial Best Paper Award in 2014, the IBM Invention Award in 2013, the KITP Rice Family Fund Fellowship in 2012, the Singapore Millennium Fellowship in 2007, and the IEEE Electron Device Society Fellowship in 2005, among other honors. $\mathrm{He}$ leads a research group in ECE that works on expanding the understanding and design of nanomaterials and nanodevices. In recent years, they have focused on the class of two-dimensional crystals and their heterostructures, and topological and magnetic materials. His team has revealed their basic electronic and optical properties, and their opportunities for novel electronics, spintronics, optoelectronics, nanophotonics, and plasmonics. He has served as a Guest Editor for Nanotechnology (IOP) and an Associate Editor for the Nanophotonics journal (De Gruyter) and Journal of Physics: Condensed Matter (IOP), as well as the Co-Chair of the "2D Materials Nanophotonics" in 2017,2019 , and 2020 .

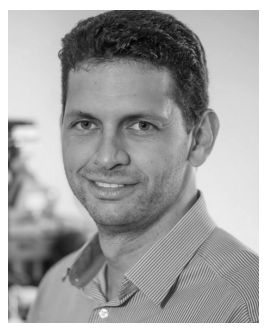

Julius Georgiou (Senior Member, IEEE) received the M.Eng. degree in electrical and electronic engineering and the Ph.D. degree from Imperial College London in 1998 and 2003, respectively. He worked as the Head of Micropower Design at Toumaz Technology, a technology-based start-up company, for two years. In 2004, he joined Johns Hopkins University as a Post-Doctoral Fellow, before becoming a Faculty Member with the University of Cyprus in 2005. He is currently an Associate Professor at the University of Cyprus. His research interests include low-power analog and digital ASICs, implantable biomedical devices, bioinspired electronic systems, electronics for space, brain-computer interfaces (BCIs), memristive devices, electronics for adaptive metamaterials, inertial and optical sensors, and related systems. He is a member of the IEEE Circuits and Systems Society and the IEEE Circuits and Systems Society Analog Signal Processing Technical Committee. He was a recipient of the Best Paper Award at the IEEE ISCAS 2011 International Symposium and at the IEEE BioDevices 2008 Conference. In 2016, he received the 2015 ONE Award from the President of the Republic of Cyprus for his research accomplishments related to the development of an "Infrared Fluorescence-Based Cancer Screening Capsule for the Small Intestine." He served as the General Chair for the 2010 IEEE Biomedical Circuits and Systems Conference, and is currently the Action Chair of the EU COST Action ICT-1401 on "MemristorsDevices, Models, Circuits, Systems and Applications - MemoCIS." $\mathrm{He}$ is also the Chair of the IEEE Biomedical and Life Science Circuits and Systems (BioCAS) Technical Committee. He has been selected as an IEEE Circuits and Systems Society Distinguished Lecturer from 2016 to 2017. $\mathrm{He}$ is also an Associate Editor of the IEEE TRANSACTIONS ON BIOMEDICAL Circuits AND Systems and the Frontiers in Neuromorphic Engineering journal. 